\title{
ENGINEERING TEAM DYNAMICS: CONNECTING FRIENDSHIP NETWORKS AND ACADEMIC TRAJECTORIES
}

\author{
Emily N. Cyr ${ }^{1}$, John Donald ${ }^{2}$, and Hilary B. Bergsieker ${ }^{1}$ \\ ${ }^{1}$ University of Waterloo; ${ }^{2}$ University of Guelph \\ encyr@uwaterloo.ca; jrdonald@uoguelph.ca
}

\begin{abstract}
Women are under-represented in STEM careers, especially engineering, as women are both less likely to enter engineering majors [1] and more likely to exit prematurely [2]. Women who leave engineering before achieving a career are then often underemployed [3]. We used an integrated research strategy to study how undergraduate classroom experiences might impact these gendered career trajectories, simultaneously modelling intrapersonal cognitions and interpersonal dynamics of students. Specifically, we studied crossdisciplinary engineering undergraduate teams over their first term in university. Importantly, this course was designed to have significant team interaction, and teams were assigned with the intention of reducing gendered barriers to participation and achievement. Our results revealed widespread gender equity (and the few observed inequities tending to shrink over the term). Further, we demonstrate specific intrapersonal and interpersonal factors that are linked to stronger academic trajectories (here operationalized as better final grades). Potential future interventions are also discussed.
\end{abstract}

Keywords: Social networks, team dynamics, academic trajectories, teamwork, gender inequity, field research, engineering, design

\section{INTRODUCTION}

Relative to men, women continue to enter engineering majors at a lower rate [1], and often exit prematurely, with only $60 \%$ of female engineering undergraduates attaining their degree versus $80 \%$ of men [2]. Although this inequity has been somewhat ameliorated in the contemporaneous Canadian context, a gender gap in STEM degree persistence remains. This attrition effectively excludes women from a highly paid career sector [4] as when women leave STEM degrees they often find themselves underemployed [3].

We propose that this gender divergence stems from a combination of intrapersonal and interpersonal factors. Specifically, the individualized stressors (e.g., selfreflection on performance and burnout) particularly associated with engineering undergraduate majors (vs. other majors) negatively impact both male and female students [5], resulting in higher exit rates from this major (vs. other majors; cite). We hypothesize that this frequently high ambient stress level combines with gender biases "in the air" [6] within mixed-gender engineering teams to produce a high-stakes STEM gender gap.

Specifically, female engineers are often stereotyped as lower in competence than their male counterparts [7], with their actual abilities underappreciated by their mentors, peers, and themselves [8], [9]. These stereotypes lead to undermining and negative interpersonal interactions with their (predominantly male) peers, directly hampering female engineers' academic trajectories [10], [11], [12]. This lack of cohesive community structure also longitudinally reduces women's abilities to handle the extra intrapersonal demands associated with engineering programs. Without peer advice- and support-based networks, women can miss out on "insider information" [13] crucial to academic success. Further, peer friendship networks can buffer against the negative socioemotional consequences associated with these rigorous academic and career-based demands [14] (e.g., higher burnout, lower confidence in abilities). As STEM women are often excluded from such beneficial networks [15], [16], [17], their academic trajectories suffer as they are less able to "bounce back" from acute (and chronic) stressors [18].

Although these distinct challenges faced by female engineering students have been well studied, scholars have typically focused on decoupled levels of analysis. Psychological researchers, for example, have often focused on intrapersonal factors leading to these gender disparities in engineering, such as implicit biases [19] or ingroup favouritism [20]. Policymakers and educational practitioners, in contrast, often highlight the structural inequities systematically disadvantaging women and how to remedy them, such as through gender-balanced admissions policies [21 and eliminating full-time study restrictions [22]. Social network analysts further study collections of relationships, such as mapping out distributed leadership structures in engineering teams [23] or wells and sinks of social capital [24]. Although all such approaches provide an important lens onto the nuanced social micro-climates within which these gender inequities emerge, we propose that interdisciplinary work covering multiple levels of analysis (e.g., individual, group, cultural) is the key antecedent to meaningful fieldlevel change. 
We propose an integrated approach to studying this phenomenon, simultaneously tracking intrapersonal cognitions and interpersonal dynamics. To do so, we longitudinally studied the experiences and academic trajectories of students in a cross-disciplinary first-year project-based engineering undergraduate course at two levels (abstract and concrete) and for two domains (academic and interpersonal). We employed analyses at both levels to disentangle holistic impressions from specific beliefs or interactions. We anticipated that although abstract experiences may be reported as positive (especially given potential self-presentational concerns in the classroom), concrete experiences may reveal hidden gendered discrepancies.

Importantly, male and female students worked for the entire term in a project team of 4-5 people but earned individual final grades (based $60 \%$ on solo work, $40 \%$ on group work). This team structure allowed us to examine student interactions over the entire term from multiple perspectives and longitudinally track student experiences. A critical factor for this particular microclimate is that these teams were intentionally formed by the instructor to reduce gender imbalances by ensuring a minimum of two women on any mixed-gender teams [25], with the hope that no woman would feel tokenized or bereft of allies within her team. Further, we ensured a heterogeneity of skills across teammates by grouping together individuals from different subdisciplines of engineering (e.g., electrical, mechanical, hydrological, biological engineering). This heterogeneity was designed to broadly "level the playing field" across team members, providing opportunity for multiple students to find their niche on the multifaceted final project.

In the results, we will first describe how perceptions of the micro-climate emerge and evolve over the course of the term for male and female students. Next, we will delineate how those gendered and subjective impressions of the micro-climate influence final grades.

\section{METHOD}

Undergraduate first-year engineering students completed baseline surveys 4 weeks after they began university (Survey 1; one lab session after meeting their teammates) and again at the end of their first term (Survey 2). Of those eligible to participate, $87 \%$ completed at least one survey $(N=333,220$ men, 107 women) and 50\% completed both surveys $(N=196,113$ men, 79 women), with lower attrition for women. Of the 81 class teams, $93 \%$ had some team network data at both timepoints ( $N=75$ teams), meaning that at least one team member completed a survey measure at the beginning and end of the term. Surveys contained both abstract (e.g., general, holistic) and concrete (e.g., specific, grounded) measures of student's academic and interpersonal experiences. Survey data were used to describe the micro-climate of the course, and to predict objective academic trajectories (operationalized here as each participant's final grade).

2.1. Objective academic trajectory. To track objective achievement, we obtained individual grades (ranging from $0 \%$ to $100 \%$ ) for the engineering course from the Registrar.

2.2. Abstract academic experiences. High-level academic burnout was measured with 12 items (e.g., "I feel burned out from my engineering studies.") and confidence in academic abilities with 8 (e.g., "I feel confident about my abilities in Guelph engineering."). Items were assessed using a scale from 1 (strongly disagree) to 7 (strongly agree).

\subsection{Concrete academic experiences.} Participants quantified their specific academic experience by realistically predicting their final course grade, on a scale from $0 \%$ to $100 \%$. In addition, participants predicted their teammates' grades (see the Appendix).

\subsection{Abstract interpersonal experiences.} General interaction quality with teammates was measured with 5 items (e.g., "I feel supported \{by my teammates\} when I face academic challenges"), comprising an overall self-centric assessment of one's relationship with the team. Further, general team cohesion (e.g. "My team sticks together better than most other design teams."), or a team-level interpretation of community connectedness, with 3 items. All items were rated from 1 (strongly disagree) to 7 (strongly agree).

2.5. Concrete interpersonal experiences. Specific team network structures were recorded via a table with all team member's names listed down rows and across columns. This table format, otherwise known in the social network analysis literature as a sociomatrix, provides a grid-like view of the relationships that are perceived to exist within a team at a particular point in time. Given our interest in mapping both social and respect-based relationships, participants completed two sociomatrices per survey, as described below.

Participants first reported who considers whom a friend using -1 (definitely does not like as a friend), 0 (may like as a friend), and 1 (definitely likes as a friend) for each directed relationship. They used a separate sociomatrix to report who respects whom as highly competent, again using -1 (definitely does not respect as highly competent), 0 (may respect as highly competent), and 1 (definitely respects as highly competent). Importantly, participants reported on their relationships toward teammates ("I see Abby as a friend") and all 
possible inter-relationships between teammates ("Abby does not see me as a friend", "Bob sees Abby as a friend").

We then assessed friendship- and respect-based indegree, or the proportion of times (out of all possible times) the participant was seen as a friend or as competent by their teammates. To quantify indegree, reports were first collapsed to 0 or 1 (-1 was selected infrequently and thus combined with 0 to indicate the absence of a friendship- or respect-based relationship), then convergence on ties with multiple reports was reached by taking first-hand reports when available (e.g., Abby's report of whether she likes Bob as a friend), and otherwise averaging the reports of all other observers (e.g., teammates' reports of whether Abby likes Bob as a friend).

Finally, for each participant, friendship- and respectbased relationships were aggregated into proportions of incoming ties (normalized indegree centrality) by teammate gender. For the friendship and respect-based social networks, each participant then had four metrics, each of which is computed as a proportion ranging from 0 to 1: female friendship indegree (e.g., friendship nominations from female teammates; Abby considers me a friend), male friendship indegree (e.g., friendship nominations from male teammates; Bob considers me a friend), female respect indegree (e.g., respect nominations from female teammates; Abby respects me as highly competent), and male respect indegree (e.g., respect nominations from female teammates; Bob respects me as highly competent).

\section{RESULTS}

\subsection{Analytic approach}

Multi-level models accounted for interdependencies between teammates' grades and grade predictions, team cohesion, interaction quality, and social network structures. However, confidence in abilities and burnout were not significantly clustered within teams, ICCs $<.14$, ps $>.358$, so multiple regression models were instead used for these two measures. Participant and teammate gender were effects-coded -1 (female) and +1 (male), and paired dummy codes tested simple effects.

\subsection{The micro-climate}

To provide a holistic description of the micro-climate within this engineering course, we examined potentially gendered student experiences at two levels (abstract and concrete) and across two domains (academic and interpersonal). The scant gender gaps in micro-climate perceptions can be seen in Figure 1. Further analyses examining predictions of teammates' grades are presented in Appendix 8.2.
3.1.1. Abstract academic experiences. At the beginning of the term, men reported significantly higher confidence in their academic abilities, $b=0.19, t(297)=$ $3.27, p=.001, d=0.38$, and marginally lower burnout, $b=-0.10, t(296)=1.88, p=.061, d=0.22$, than women. However, these gender differences faded over time, with no differences between male and female participants' confidence or burnout, $p s>.236$, at the end of the term (controlling for baseline measures).

3.1.2. Concrete academic experiences. Women received significantly higher final grades $(M=83.20 \%)$ than men $(M=79.32 \%), b=-1.99, t(290.35)=4.68, p<$ $.001, d=0.55$. When predicting their own final grades at the beginning of the term, men and women diverged in accuracy, $b=2.06, t(271.09)=3.29, p=.001, d=0.40$. Men overestimated their academic achievements, reporting a predicted grade about $4.41 \%$ higher than their actual final grade. In contrast, women made highly accurate judgements, with their guess non-significantly differing from their actual final grades. By the end of the term (controlling for initial guesses), this gender gap in accuracy shrunk, $b=0.67, t(177.08)=1.50, p=.137, d$ $=0.22$, although men were still slightly overestimating their final grades.

\subsubsection{Abstract interpersonal experiences.} Perceived team cohesion and overall team interaction quality did not differ by participant gender at either timepoint, $p s>.194$, with men and women reporting equivalently positive abstract interpersonal microclimates.

3.1.4. Concrete interpersonal experiences. Granular measures of interpersonal experiences were captured via two sociomatrices (one for friendship ties and one for respect ties).

3.1.4.1. Friendship networks. At the beginning of university, women (vs. men) were quickly considered a friend by a higher proportion of their female teammates, $b=-0.07, t(210.82)=2.49, p=.014, d=0.34$. This gender gap in incoming friendship ties from women was exacerbated over the course of the term, with women being befriended by female teammates more rapidly than men were (controlling for baseline friendships with women $), b=-0.10, t(193.30)=3.91, p<.001, d=0.56$. Incoming friendship ties from male teammates followed a similar, but attenuated pattern to those from female teammates. Namely, men had marginally higher male friendship indegree than women $\mathrm{did}, b=0.04, t(311.38)$ $=1.68, p=.094, d=0.19$, and were nominated by new male teammates members marginally more quickly (controlling for baseline nominations) than women were, $b=0.04, t(281.84)=1.94, p=.054, d=0.23$. 
3.1.4.2. Respect networks. Converse to the friendship networks, women and men were equally likely to be respected by a high proportion of their female teammates, $b=-0.03, t(209.65)=1.18, p=.239, d=0.16$. A gender gap, however, again emerged in incoming ties from female teammates, $b=-0.06, t(189.34)=2.47, p=.014$, $d=0.36$ (controlling for baseline friendships), with men (vs. women) respected by an increasingly smaller proportion of their female teammates. Baseline respect from male teammates was equitable, $b=0.00, t(304.19)$ $=0.22, p=.826, d=0.03$, and did not vary over the term for either men or women, $b=-0.01, t(285.55)=0.39, p=$ $.697, d=0.05$.

\subsection{How the micro-climate influences grades}

The results presented below identify the impact each of the key survey measures have on final grades. The strength of these findings can be seen in Figure 2, which shows the effect size (Cohen's $d$ ) of average measures (mean of Survey 1 and Survey 2) versus the changes in the measures from the beginning to the end of the term (Survey 2 minus Survey 1), when tested simultaneously. All models were tested for gender moderation (significantly different effects for men versus women) and unless noted did not differ by gender.

3.2.1 Abstract academic experiences. We simultaneously model average (mean of Survey 1 and 2) and changing (Survey 2 minus Survey 1) confidence in abilities, as well as the respective gender moderation terms, on final grades. Only the change term predicted final grades, $b=1.20, t(157.72)=2.07, p=.040, d=$ 0.33 , meaning that improving perceptions of one's academic abilities is linked to better final grades (irrespective of mean-level confidence). However, average confidence in abilities was not linked to final grades, $b=-0.45, t(150.40)=0.87, p=.385, d=0.14$, such that those maintaining high confidence did no better in the course than those feeling less confident.

The parallel model for burnout revealed the same pattern, $b=-1.43, t(157.69)=2.25, p=.026, d=0.36$, again with decreasing burnout associated with better final grades (regardless of average burnout over the term). Additionally, maintaining low burnout did not improve grades, $b=-0.34, t(144.74)=0.67, p=.502, d$ $=0.11$.

3.2.2. Concrete academic experiences. Average and changing grade prediction error was also regressed onto actual final grades (with the respective gender moderation terms). Increasingly accurate grade predictions were associated with better final grades, $b=$ $0.22, t(146.70)=4.43, p<.001, d=0.73$.

3.2.3. Abstract interpersonal experiences. Models predicting final grades using average versus change scores revealed that team cohesion was decoupled from final grades, with consistently high and improving team cohesion not predicting better or worse final grades, $p s>.650$. However, interaction quality mirrored the effects seen for confidence in abilities and burnout. Namely, improving in interaction quality with one's teammates was significantly tied to better final grades, $b$ $=1.38, t(159.24)=3.59, p<.001, d=0.57$, again despite a lack of linkage to average interaction quality, $b=0.05$, $t(167.01)=0.08, p=.933, d=0.00$.

\subsubsection{Concrete interpersonal experiences.} Friendship and respect networks were both, but distinctly, related to final grades, as outlined below. The beneficial effects of network structures again did not differ significantly by gender, with women and men once again equivalently benefiting.

3.2.4.1. Friendship networks. We then simultaneously tested the impact of persistent and changing friendships on final grades (as well as gender moderation). Being nominated by an increasing proportion of female teammates over time predicted better final grades, $b=1.05, t(173.92)=2.59, p=.010, d$ $=0.39$, regardless of one's average female indegree, $b=$ $0.04, t(177.05)=0.07, p=.948, d=0.01$, which was not linked to final grades. In contrast, being consistently liked by a high proportion of male teammates predicted higher grades, $b=1.70, t(270.94)=3.12, p=.002, d=0.38$, although increasing in male indegree did not, $b=0.46$, $t(242.88)=1.18, p=.240, d=0.15$.

3.2.4.2. Respect networks. As with our examination of friendship networks, we tested the impact of persistent and of changing respect indegree on grades. Regardless of target or origin gender, maintaining high respect centrality (and improving over time) was linked to higher grades. Namely, high average respect from women, $b=$ $1.72, t(179.21)=3.08, p=.002, d=0.46$, and men, $b=$ $1.84, t(275.60)=3.50, p<.001, d=0.42$, as well as increasing respect from women, $b=1.13, t(166.77)=$ $2.94, p=.004, d=0.46$, and men, $b=1.21, t(253.53)=$ $3.59, p<.001, d=0.45$, were equivalently positively linked to higher final grades. 


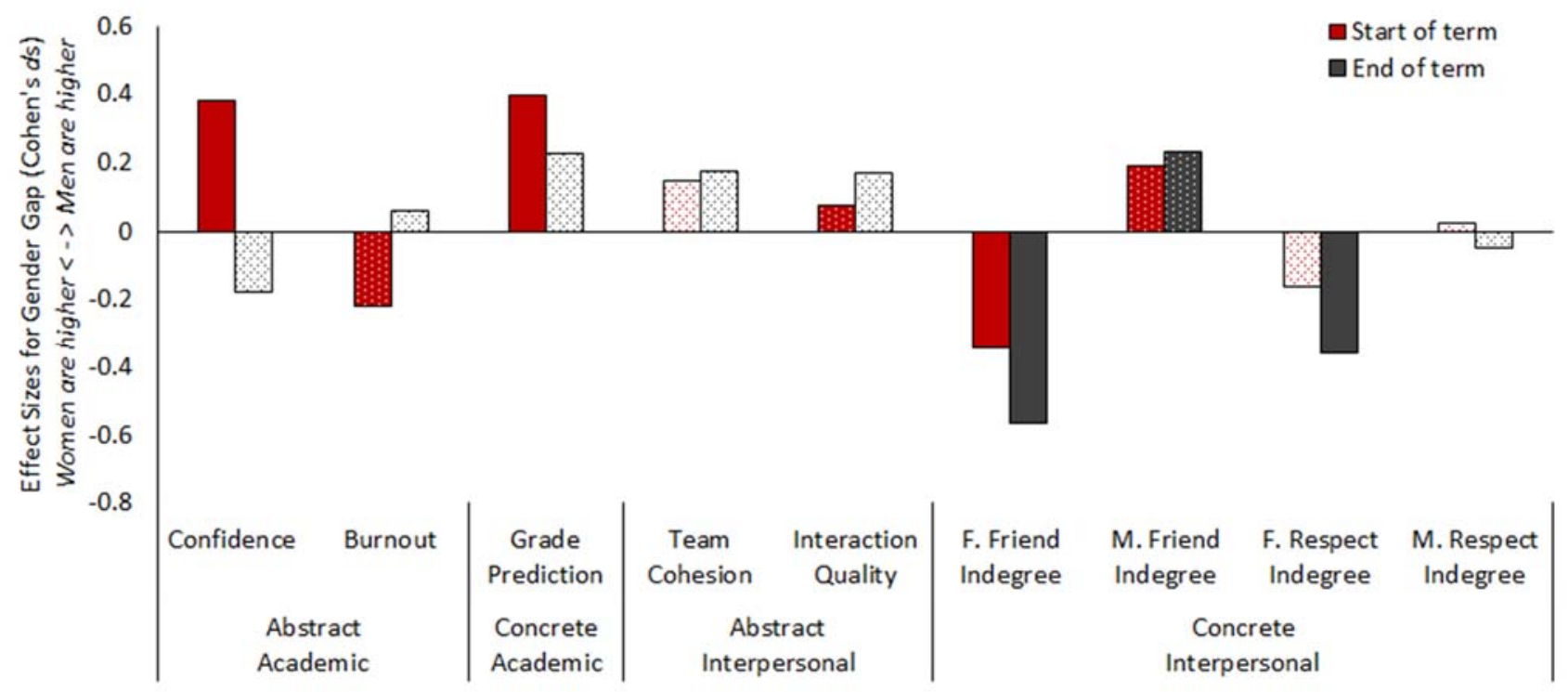

Figure 1. Gendered Perceptions of the Micro-climate. Effect sizes (Cohen's ds) of gender gaps on each of our primary measures, at the beginning of the term and at the end of the term (statistically controlling for start of term values). Positive values indicate gender gaps when men reported higher values than women, and negative values indicate measures upon which women were higher than men. Solidly filled bars indicate significant effects, $80 \%$ dotted bars marginal effects, and $20 \%$ dotted bars non-significant effects. "F." indicates nominations from female teammates and "M." from male teammates.

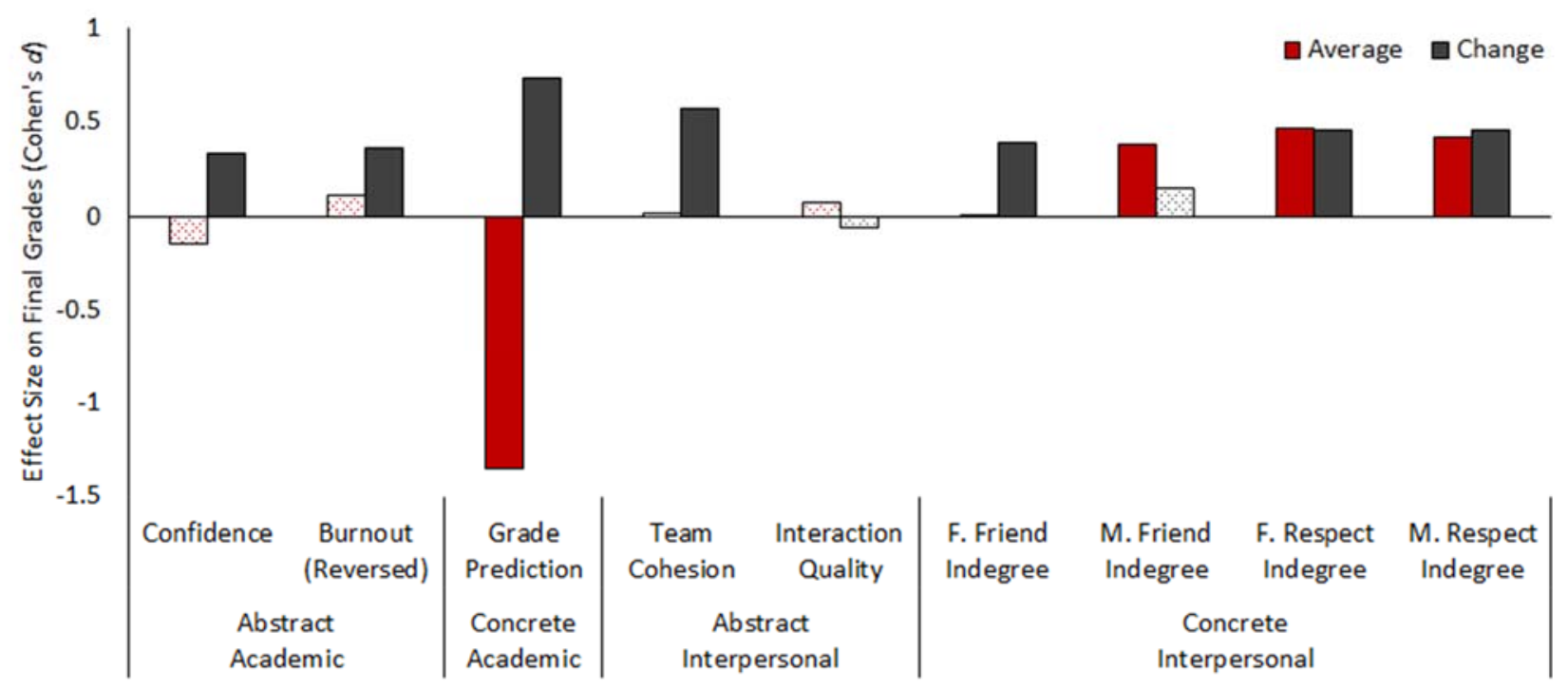

Figure 2. Influence of Micro-climates on Grades. Effect sizes (Cohen's ds) for the average and change scores of our primary measures on actual final grades. Positive values indicate a positive relationship between that measure and final grades, and negative values indicate a negative relationship. Solidly filled bars indicate significant effects, and bars with a $20 \%$ dotted pattern fill indicate non-significant effects." $F . "$ indicates nominations from female teammates and "M." from male teammates. 


\section{DISCUSSION}

Using a multi-method approach, we explored how student experiences (captured by quantitative social network and psychological survey methodology) influence final grades. The results reflect the unique policies and team dynamics in the micro-climate of this first-year project-based engineering design course. We discuss the specific benefits afforded to female engineers in this carefully constructed environment aimed at reducing gendered barriers, and describe specific levers future educators may use to improve their microclimates.

\subsection{Continually improving confidence and reducing burnout.}

In our sample, as in many engineering environments, men had better abstract academic experiences (reporting stronger academic confidence and lower burnout) at the beginning of the term. However, potentially as a result of our class structure, this gender gap disappeared once male and female students settled into their coursework and project teams. Abstract academic experiences also had concrete consequences: Improvements were linked with better final grades. For example, women who gained confidence over the course of the term achieved significantly above-average final grades (yet men who reported consistently high confidence did not).

This linkage hinging on changing experiences, rather than more consistent individual differences, reveals a potential avenue for further improving grades. Namely, class policies aimed at actively boosting confidence and reducing burnout may lead to real gains in student grades. These effects were seen across the continuum of averaged experiences, meaning that regardless of initial confidence or burnout, improvements are likely to be beneficial. Specifically, even if Alice starts with relatively low burnout, further reducing her burnout may still improve her grades.

\subsection{Accurately predicting final grades.}

Women accurately perceived their concrete academic outcomes, predicting they would achieve higher final grades than their male counterparts. Men instead overestimated their final grades when making predictions at the beginning of the term, but grew somewhat more accurate over time. Further, those who became more accurate tended to achieve higher final grades. More frequent grade distribution, or a stronger encouragement of students to deeply process their grades, may be a simple and low-cost strategy. However, the fairly large contingent of students who made consistent grade overestimations (53\%) despite the (relatively frequent) grade distribution schedule may require additional intervention.

\subsection{Strengthening team cohesion.}

Team cohesion and interaction quality among teammates did not significantly differ by participant gender. However, improvements in team cohesion over the term were associated with both men and women receiving higher final grades. One mechanism plausibly driving this effect is improved advice-seeking and distribution of labour within the team, as a result of deepening communication and goal coordination. Future interventions focusing on building team cohesion over the term (rather than focusing on initial levels) may be warranted. Conversely, improving general interaction quality among teammates is not predicted to be a fruitful avenue toward improving student grades.

\subsection{Women's changing beliefs, and men's consistency.}

Friendship nominations made by women tended to be relatively homophilous, with women (vs. men) receiving significantly more friendship nominations from female teammates, and this gap increasing over time. Women also tended to lose respect for male teammates over the term, despite maintaining respect for female peers. This flux in relationships originating from women shows that continued intervention may plausibly improve women's friendship and respect networks, as women tend to update their relationships with greater regularity (although these updates tend toward making their networks more gender homophilous).

In contrast, men were relatively unchanging in their nominations over time: If a male teammate liked or respected a teammate at the onset of the term, he was quite likely to also do so at the end. Further, men in our sample tended to report liking and respecting an equivalent proportion of their male and female teammates. This stands in contrast to work by many scholars showing men (like women) tend to befriend and respect more same-gender peers [26]. As men tend to "pick and stick" with teammates (in terms of seeing them as a friend or respecting them as highly competent), initial team-building exercises may form a critical component of healthy relationships stemming from male engineers, toward both men and women.

Such network structures critically bear on final grades, with those highly respected by their teammates (of both genders) achieving the highest final grades. The proposed interventions boosting women's continuous befriending of teammates, and maintaining men's positive first impressions, may therefore further improve academic trajectories. 


\section{CONCLUSIONS}

Fostering equitable environments within our curriculum necessitates looking for ways to quantify the impact of our efforts. This research project leverages approaches from multiple disciplines, blending grounded educational expertise with quantitative social network and psychological methods to push forward our understanding of the distinct contributions of individual beliefs and team dynamics on academic trajectories. Through exploring the unique policies of this particular micro-climate, we illuminate the general and genderspecific benefits afforded to female engineering students in a carefully constructed environment aimed at reducing gendered barriers. Further, we reveal how individual cognitions and interpersonal structures link to grades, illuminating specific levers upon which future educators can push. For example, activities that boost confidence and reduce burnout over the course of the semester correlate with higher final grades for women, regardless of the starting level. Similarly, improvements in team cohesion correlate with higher final grades for women as well as for men, regardless of the starting levels. From a team dynamics perspective, having more women see you as a friend over the semester correlated to higher final grades for both men and women.

This initial study provides encouraging initial evidence that facilitating an environment of gender equity within an undergraduate classroom environment with significant team interaction appears to create positive academic outcomes for both women and men in engineering. We hope to leverage these findings to look for ways of continuing to improve the classroom environment from the perspective of both gender equity and academic success for all.

\section{ACKNOWLEDGEMENTS}

This work was supported by SSHRC Grant \#8952017-1025. We also thank collaborators Mary Wells and Tyler Hartwig for their project support and feedback.

\section{REFERENCES}

1 J. Jorstad, S. S. Starobin, Y. Chen, \& A. Kollasch. "STEM aspiration: The influence of social capital and chilly climate on female community college students." Community College Journal of Research and Practice, vol. 41, no. 4-5, pp. 253-266, 2017.

2 A. E. Bell, S. J. Spencer, E. Iserman, \& C. E. Logel. "Stereotype threat and women's performance in engineering." Journal of Engineering Education, vol. 92, no. 4, pp. 307-312, 2003.

3 X. Chen. "STEM Attrition: College Students' Paths Into and Out of STEM Fields (NCES 2014-001)". National Center for Education Statistics, Institute of Education Sciences, U.S. Department of Education. Washington, DC., 2013.
PayScale. "Majors that pay you back." 2013. Retrieved from http://www.payscale.com/

5 G. Lichtenstein, A. C. McCormick, S.D. Sheppard, \& J. Puma. "Comparing the undergraduate experience of engineers to all other majors: Significant differences are programmatic." Journal of Engineering Education, vol. 99, no. 4, pp. 305-317, 2010.

6 C. M. Steele. "A threat in the air: How stereotypes shape intellectual identity and performance." American Psychologist, vol. 52, no. 6, pp. 613-629, 1997.

7 C. Logel, G. M. Walton, S. J. Spencer, E. C. Iserman, W. von Hippel, \& A. E. Bell. "Interacting with sexist men triggers social identity threat among female engineers." Journal of Personality and Social Psychology, vol. 96, no. 6, pp. 1089-1103, 2009.

8 C. Logel, E. C. Iserman, P. G. Davies, D. M. Quinn, \& S. J. Spencer. "The perils of double consciousness: The role of thought suppression in stereotype threat."

Journal of Experimental Social Psychology, vol. 45, no. 2, pp. 299-312, 2009.

9 G. M. Walton \& S. J. Spencer. "Latent ability: Grades and test scores systematically underestimate the intellectual ability of negatively stereotyped students." Psychological Science, vol. 20, no. 9, pp. 1132-1139, 2009.

10 T. Schmader, M. Johns, \& C. Forbes. “An integrated process model of stereotype threat effects on performance." Psychological Review, vol. 115, no. 2, pp. 336-356, 2008.

11 S. J. Spencer, C. Logel, \& P. G. Davies. "Stereotype threat." Annual Review of Psychology, vol. 67, no. 1, pp. 415-443, 2016.

12 S. J. Spencer, C. M. Steele, \& D. M. Quinn. "Stereotype threat and women's math performance." Journal of Experimental Social Psychology, vol. 3, no. 1, pp. 4-28, 1999.

13 A. Morgan, J. Quesenberry, \& E. Trauth. "Exploring the importance of social networks in the IT workforce: Experiences with the 'boy's club'.” AMCIS 2004 Proceedings, pp. 1313-1320, 2004.

14 P. M. Sias \& D. J. Cahill. "From coworkers to friends: The development of peer friendships in the workplace." Western Journal of Communication, vol. 62, no. 3, pp. 273-299, 1998.

15 K. M. Bartol \& X. Zhang. "Networks and leadership development: Building linkages for capacity acquisition and capital accrual." Human Resource Management Review, vol. 17, no. 4, pp. 388-401, 2007.

16 M. L. Forret \& T. W. Dougherty. "Networking behaviors and career outcomes: Differences for men and women?" Journal of Organizational Behavior, vol. 25, no. 1, pp. 419-437, 2004.

17 J. Wang. "Networking in the workplace: Implications for women's career development." New Directions for Adult and Continuing Education, vol. 122, no.1, pp. 3342, 2009.

18 S. E. Wilks \& C. A. Spivey. "Resilience in undergraduate social work students: Social support and adjustment to academic stress." Social Work Education, vol. 29, no. 3, pp. 276-288, 2010. 
19 E. N. Cyr, H. B. Bergsieker, T. C. Dennehy, \& T. Schmader. "Old boys' network: Social exclusion of women reflects implicit bias and predicts career constraints." Manuscript in preparation.

20 D. R. Smith, N. DiTomaso, G. F. Farris, \& R. Cordero. "Favoritism, bias, and error in performance ratings of scientists and engineers: The effects of power, status, and numbers." Sex Roles, vol. 45, no. 5-6, pp. 337-358, 2001.

21 B. M. Holloway, T. Reed, P. K. Imbrie, \& K. Reid. "Research-informed policy change: A retrospective on engineering admissions." Journal of Engineering Education, vol. 103, no. 2, pp. 274-301, 2014.

22 K. Beddoes. "Selling policy short? Faculty perspectives on the role of policy in addressing women's underrepresentation in engineering education." Studies in Higher Education, vol. 43, no. 9, pp. 1561-1572, 2018.

23 Q. Wu \& K. Cormican. "Shared leadership and team creativity: A social network analysis in engineering design teams." Journal of Technology Management \& Innovation, vol. 11, no. 2, pp. 2-12, 2016.

24 J. Skvoretz, G. Kersaint, R. Campbell-Montalvo, J. D. Ware, C. A. S. Smith, E. Puccia, J. P. Martin, R. Lee, G. MacDonald, \& H. Wao. "Pursuing an engineering major: social capital of women and underrepresented minorities." Studies in Higher Education, vol. 2019, pp. 1-16, 2019.

25 B. Oakley, R. Brent, R. M. Felder, and I. Elhajj. "Turning Student Groups into Effective Teams." Journal of Student Centered Learning, p. 9-34, 2004.

M. McPherson, L. Smith-Lovin, \& J. M. Cook. "Birds of a feather: Homophily in social networks." Annual Review of Sociology, vol. 27, no. 1, pp. 415-444, 2001.

\section{APPENDICES}

\subsection{Statistical terms.}

$b$ Direction of the relationship. Slope of the relationship between the predictor variable and the dependent variable. Positive slopes indicate a positive correlation, and negative slopes indicate an inverse relationship. This metric is not standardized, so the magnitude can only be visually compared if the dependent variables are on the same scale.

$p \quad$ Significance of the relationship. Metric indicating the significance of the relationship between the predictor variable and the dependent variable. Relationships are deemed "statistically significant" if $p<.05$, "marginally significant" if $.1<p<.05$, and non-significant otherwise.

$d \quad$ Standardized size of the relationship. Cohen's $d$, or simply $d$, is a measure of the size of the difference between groups, and is standardized so that you can compare it across different measures, models or even studies.

Conventionally, a small effect is seen as $d=0.2$, a medium effect as $d=0.5$, and a large effect as $d=0.8$.

\subsection{Predicting teammate's grades.}

End-of-term predictions regarding teammates' grades revealed a parallel asymmetry: Participants accurately predicted female teammates' grades, $b=0.84, t(524.82)$ $=1.57, p=.118, d=0.14$, with both men and women making well-tuned predictions about women, $b=0.08$, $t(524.82)=0.14, p=.887, d=0.01$. Yet participants systematically overestimated male teammates' grades, $b$ $=2.41, t(553.20)=6.21, p<.001, d=0.53$, especially men, $b=1.45, t(553.20)=3.74, p<.001, d=0.32$.

Taken together, participants (especially women) more accurately perceived women's grades (their own grades or teammate's grades). Conversely, participants (especially men) tended to overestimate men's grades. Grade prediction error for teammates is presented in Figure 3.

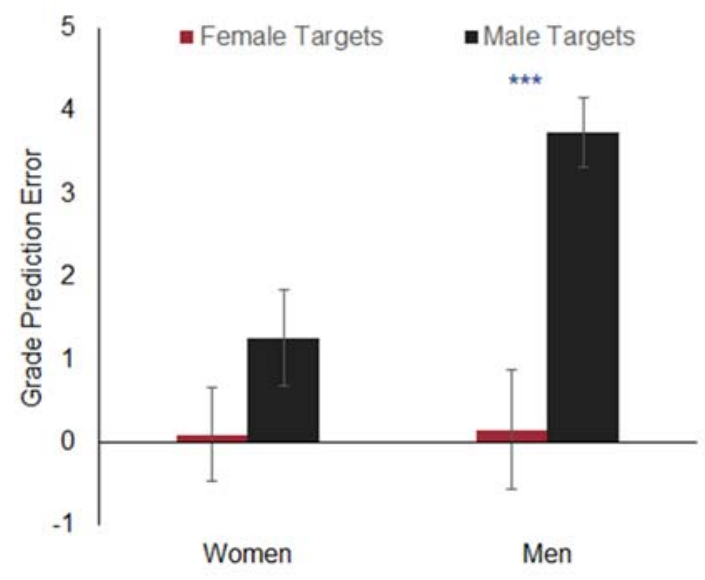

Figure 3. Prediction error for teammates' grades by participant and teammate gender 